\title{
Fast and effective: Intuitive processes in complex decisions
}

\author{
Michael Brusovansky ${ }^{1} \cdot$ Moshe Glickman $^{1} \cdot$ Marius Usher $^{1}$ \\ Published online: 27 April 2018 \\ (C) Psychonomic Society, Inc. 2018
}

\begin{abstract}
Is it possible to carry out complex multi-attribute decisions (which require an estimation of the weighted average) intuitively, without resorting to simplifying heuristics? Over the course of 600 trials, 26 participants had to choose the better-suiting jobcandidate, a task requiring comparison of two alternatives over three/four/five dimensions with specified importance weights, with a time constraint forcing intuitive decisions. Participants performed the task fast (mean reaction time (RT) $\sim 1.5 \mathrm{~s})$ and with high accuracy $(\sim 86 \%)$. The participants were classified as users of one of three strategies: Weighted Additive Utility (WADD), Equal Weight rule and Take-The-Best heuristic (TTB). Fifty-nine percent of the participants were classified as users of the compensatory WADD strategy and 29\% as users of the non-compensatory TTB. Moreover, the WADD users achieved higher task accuracy without showing time costs. The results provide support for the existence of an automatic compensatory mechanism in weighted average estimations.
\end{abstract}

Keywords Decision making $\cdot$ Weighted average $\cdot$ Compensatory process $\cdot$ Take-the-best

\section{Introduction}

Complex decisions, such as selecting a job candidate or a vacation package, are among the most demanding and challenging human activities. A major cause of this challenge is the presence of trade-offs between attributes (e.g., intelligence vs. motivation for job candidates) that are difficult to compare. While a normative theory, based on weighted additive utility $(W A D D)$, was developed by early decision theorists (Keeney \& Raiffa, 1976), a widely accepted view considers that the computations required for the normative WADD algorithm are too complex for online human decisions (not assisted by offline calculations or external aids). Accordingly, it is often

Electronic supplementary material The online version of this article (https://doi.org/10.3758/s13423-018-1474-1) contains supplementary material, which is available to authorized users.

Michael Brusovansky

brusovan@post.tau.ac.il

Marius Usher

marius@post.tau.ac.il

1 School of Psychological Sciences and Sagol School of Neuroscience, Tel-Aviv University, Tel-Aviv, Israel assumed that when faced with such decisions, humans typically resort to a number of simplifying non-compensatory heuristics, such as Take-the-Best (TTB), according to which one chooses on the basis of the most important attribute (in case of a tie, the second most important attribute is considered; Gigerenzer \& Goldstein, 1996, 1999; Payne, Bettman, \& Johnson, 1993; but see Newell, 2005, for a critique of this approach and suggestions of formal models of ecological rationality). Such heuristics simplify the decision algorithm, by replacing the compensatory processes - in which all the attributes are weighted into the decision - with a noncompensatory one, in which only a small subset of the attributes is taken into account (Dieckmann \& Rieskamp, 2007; Gigerenzer \& Goldstein, 1999; Tversky, 1969, 1972).

Recent research has challenged the assumption that compensatory strategies are too complex and thus beyond daily decision-making ability. First, numerous studies in the domain of probabilistic inference with binary cues have shown that even when environments are designed to promote the use of TTB heuristic, a significant proportion of participants do not "take the best" (e.g., Bröder, 2000; Lee \& Cummins, 2004; Newell \& Shanks, 2003). Second, more recent experimental work has demonstrated that most participants make probabilistic inferences based on multiple cues in a compensatory yet rapid and automatic manner (Glöckner \& Betsch, 2008, 2012; Glöckner, Hilbig, \& Jekel, 2014). Other research has 
manipulated time pressure, confirming the presence of compensatory strategies with a 3-s response-deadline and, for some participants, even for a strict deadline of $750 \mathrm{~ms}$ (Oh et al., 2016).

A mechanistic account of such an automatic yet compensatory decision process was proposed by Glöckner and colleagues in the form of the PCS model (Glöckner et al., 2014). PCS is a connectionist, accumulator-type model, that integrates (using a parallel architecture) differences in weighted evidence $(\Delta \mathrm{WA})$ between the alternatives and predicts slower reaction times (RTs) for decisions with smaller $\triangle \mathrm{WA}$ (Glöckner \& Betsch, 2008, 2012; see also Roe, Busemeyer, \& Townsend, 2001 for an accumulator-type Decision-Field-Theory model of multiattribute decisions with similar RT predictions).

In this paper we demonstrate an ability to make complex decisions using compensatory, rapid, and automatic mechanisms in a different domain: multi-attribute decision-making based on numerical (non-binary) attributes. Such decisions normatively require a weighted averaging computation, traditionally associated with analytical processes. Moreover, multiattribute decisions with non-binary attributes have received less attention in recent research (see Russo \& Dosher, 1983 and Tversky, 1969 for some older studies) and they differ from binary cue decisions in a number of important aspects (problem-space is virtually infinite and precludes the use of simplifying strategies, such as memorizing or counting). Therefore, if rapid and compensatory (WADD) strategies can be deployed in this domain, this would provide support for the impressive power of the intuitive decision-maker.

Recent research has shown that an important precursor of $W A D D$ - numerical averaging - can be estimated in a relatively precise and yet automatic manner (Brezis, Bronfman, Jacoby, Lavidor, \& Usher, 2016; Brezis, Bronfman, \& Usher, 2015; Rusou, Zakay, \& Usher, 2017). Here we set to test whether this ability extends to weighted averaging, by employing a job selection multi-attribute decision task.

\section{Experiment}

Participants were asked to take the role of a job interviewer who chooses one of two candidates based on the candidates' abilities on several attributes and their relative importance. We varied (in blocks) the number of attributes (three/four/five), and we presented a large set of decision problems with randomized values (see Methods). This design allows us to contrast decision strategies within each participant using choices and RTs. While the TTB heuristic predicts slower decisions in cases there is a tie on the most important attribute, PCS (or other accumulator models) predicts decision times that increase with lower $\Delta \mathrm{WA}$. Another central question of interest is whether the deployment of compensatory strategies results in improved task performance.

\section{Method}

\section{Participants}

Twenty-six students from Tel-Aviv University (14 females, age: $19-31, M=24.7)$ participated in the experiment, in exchange for course credit and payment that was dependent on performance. On average, participants received 30 NIS $(\sim 7.5$ USD). The sample size was set at 26 with each subject tested in three tasks, allowing for $78(26 \times 3)$ classifications in total to be made (see Strategy Classifications section).

\section{Materials}

Each decision was presented in a table-format (see Table 1). Three jobs were presented, with three, four, and five attributes, respectively. Each job specified the attributes' importance (i.e., weight; see Table 1). When the job had three attributes, the specified importance-weights were 3, 2, and 1 (i.e., the most important attribute was three times more important than the least important attribute), for the four-attribute job they were $4,3,2$, and 1 , and for the five-attribute job 5, 4, 3, 2, and 1 . The values the candidates received in each trial were generated randomly, as random integers between 1 (poor) and 9 (excellent; from a uniform distribution; if the resulting weighted average for the two candidates was the same, the ratings were generated anew).

A time limit for providing an answer was imposed, in order to encourage participants to rely on their intuitive mind-set and not explicitly compute weighted averages (Horstmann, Hausmann, \& Ryf, 2010). The time limit increased with the number of attributes that had to be considered to make sure that all the information can be encoded. The time limits were $3 \mathrm{~s}$ for the 3 -attributes, $4 \mathrm{~s}$ for the 4 -attributes, and $5 \mathrm{~s}$ for the 5 attribute jobs. As we report below, however, the time constraints did not affect the actual decision times.

\section{Procedure}

Participants completed 600 trials overall, with three blocks of 200 trials for each job (3, 4, or 5 attributes). On each trial, a choice problem (see Table 1) was presented until the participant entered a decision by using the keyboard. Visual feedback (correct/incorrect) was given after each trial, based on the weighted averages. Feedback was also given on the number of correct trials the participant accumulated, which was translated to monetary reward at the end of the experiment. Once the time limit expired, the trial ended, and the visual feedback, ("too slow," was presented. The whole procedure took approximately $60 \mathrm{~min}$ (see Supplementary Material for details). 
Table 1 Example of a trial in which the job had four attributes, with weights of 4, 3, 2, and 1 . Here candidate A had the higher weighted average ( 5.2 vs. 4.3 for candidate $B$ ) and so she should be selected for the job, while candidate B should be selected according to TTB heuristic

\begin{tabular}{lll}
\hline & A & B \\
\hline Intelligence -4 & 3 & 3 \\
Work Ethic -3 & 5 & 7 \\
Easy to Work With -2 & 9 & 4 \\
Creativity -1 & 7 & 2 \\
\hline
\end{tabular}

\section{Results}

\section{Group analyses}

\section{Accuracy}

To test effects of difficulty and task practice (defined across four chunks of 50-trial sub-blocks) on task accuracy, a repeated-measures ANOVA was carried out, with number of attributes (three/four/five) and sub-block as within-subject factors. As shown in Fig. 1 (solid lines), there was a main effect of difficulty $(F(2,50)=37.39, p<.001)$. As the number of attributes increased accuracy dropped from $90 \%$ for the three attributes to $86 \%$ for four attributes and $84 \%$ for five attributes. No main effect of sub-block emerged $(F(3,75)=1.16, p=.329)$, indicating that extensive learning is not necessary. For each number of attributes, the taskaccuracy was higher than a bound obtained from an errorless version of the TTB heuristic (dashed lines), $F(1,25)=74.29, p<.001$.

\section{Reaction times}

Mean RT and the average number of trials (out of 200) in which the time limit was missed are given in Table 2 .

A repeated-measures ANOVA, with number of attributes (three, four, five) as within-subject factor, revealed no effect of number of attributes, $F(1.25,31.23)=0.056, p=.865$. Thus, although the task's difficulty increased and more information had to be considered, participants did not require more time. The number of trials in which the time limit was missed was only $0.6 \%$ of all trials and the average decision time was of around 1.5 s (see also Glöckner \& Betsch, 2008, for similar results).

We also examined the correlation between RT and task accuracy. For each participant, we calculated the correlations between RT and accuracy, across all trials, separately for each number of attributes. The resulting mean correlations were negative, as predicted by the automatic WADD mechanism: $r=-0.199$ for three attributes, $r=-0.203$ for four attributes and $r=-0.172$ for five attributes. Interestingly, the negative correlations between accuracy and RT remained even after controlling for the trials' difficulty: the partial correlation between RT and accuracy remained negative: $r=-0.102$ for three attributes, $r=-0.084$ for four attributes, and $r=-0.062$ for five attributes. While these partial correlations are small, they are all significantly different from 0 (all $p s^{\prime}<.05$, tested using a bootstrap procedure with 10,000 resamples) and in the same direction, suggesting that taking longer to decide reduces accuracy.

\section{Strategy classifications}

We next examined individual differences in decision strategies. We tested three potential strategies that participants could use when performing the task: the weighted average $W A D D$, $T T B$, and the Equal Weights rule combined with the TTB heuristic $(E Q W-T T B)$. According to the $E Q W-T T B$ strategy, one chooses the alternative for which the non-weighted average is highest (i.e., the subjects average the values but ignore the importance weights). In cases where the non-weighted average for both alternatives is the same, participants choose according to the TTB heuristic, thus its name $-E Q W-T T B .{ }^{1}$

We start using a simplified "trembling-hand" approach (Bröder, 2010), according to which the subject has a probability $p$ to mistakenly report an alternative not predicted by the choice strategy. We use this approach in order to obtain an upper bound on the proportion of TTB use (we defer to the computational section, where we examine a more refined type of strategy-classifications allowing probabilistic errors and strategy mixtures).

The classifications were done separately for three/four/five attributes to test whether increased difficulty leads to more reliance on non-normative strategies. To classify the participants based on the three strategies - WADD, TTB, and EQW$T T B$ - we computed the probability of the data ( 200 choices) for each strategy and we selected the strategy that has the highest probability; see Supplementary Material for details of the classification procedure. The classification results are shown in Table 3 (see Table S1 in Supplementary Material for individual classifications and Tables S2, S3, and S4 for the normalized probabilities of the three strategies); $82 \%$ of the classifications (64 out of 78) are associated with normalized probability larger than .99 , and $88 \%$ (69 out of 78 ) with a probability larger than .90 .

As shown in Table 3, the majority of classifications (46 out of 78 in total, $\sim 59 \%$ ) belong to the compensatory (normative) $W A D D$ strategy and another $8 \%$ (six in total) were a less optimal but still compensatory EQW-TTB strategy. Only $29 \%$ of the classifications (23 in total) fell into the non-compensatory

\footnotetext{
${ }^{1}$ We also tested a less restrictive Take-Two heuristics (Dieckmann \& Rieskamp, 2007), but none of the data sets were classified to this strategy and therefore we do not include it in Table 3.
} 


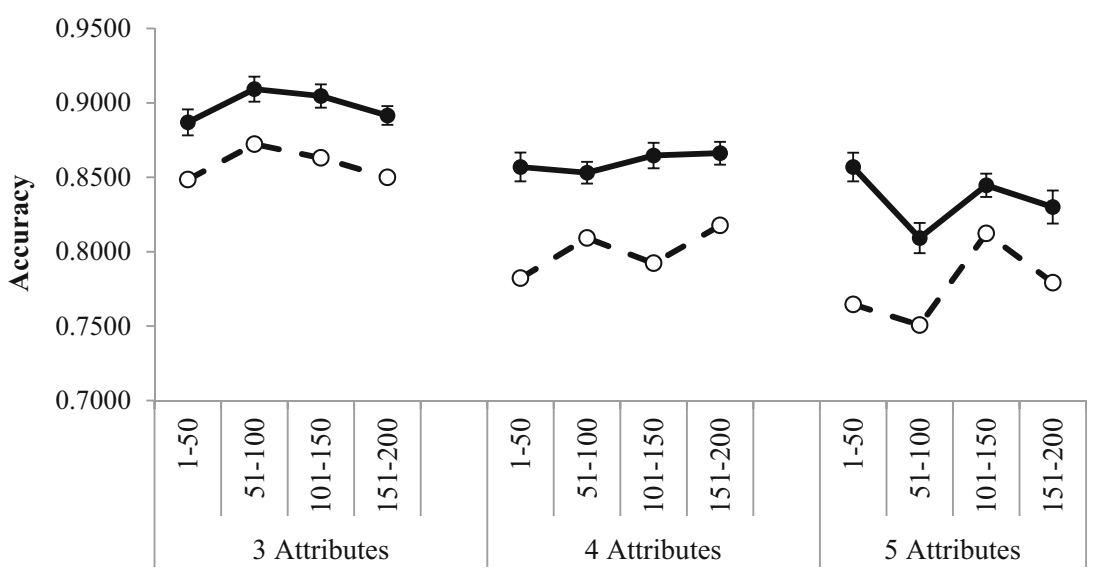

Fig. 1 Task-accuracy. Solid-lines: accuracy as a function of the number of attributes and of trial-number (in 50-trial blocks). Dashed lines: theoretical performance of TTB heuristic. Error bars represent within-subject standard errors (Cousineau, 2005)

TTB category. The amount of WADD classifications did not vary with the number of attributes. A summary of participants' accuracy as a function of strategy is shown in Fig. S1 (see Supplementary Material). We find that users of the WADD strategy had higher accuracy than TTB users, $t(67)=3.08$, $p=.003$. As reported in the Supplementary Material, this is not due to a speed-accuracy tradeoff.

\section{Attributes' weights}

Using logistic regression, we computed the subjective weights each participant gave to each of the attributes. Figure 2 shows these subjective weights for the three strategy subgroups (see Fig. S2 in the Supplementary Material for the group weights). These weights indicate that $W A D D$ users are better calibrated with the objective weights, the results of the TTB users show a strong overestimation of the most important attribute, confirming their reliance on a single dimension. A repeatedmeasures ANOVA on the attributes' weights of users of the $T T B$ and WADD strategies revealed an interaction between the strategy used and the attributes' weights, for every number of attributes - for three attributes: $F(2,42)=21.36, p<.001$, for four attributes: $F(3,63)=13.93, p<.001$, and for five attributes: $F(4,92)=15.40, p<.001$. The $E Q W-T T B$ users showed the flattest curves, consistent with the equality of weights characterizing this strategy.

Table 2 Mean reaction time (RT) (standard deviations in parentheses) and average number of trials in which the time limit was missed (out of 200 ), for each number of attributes

\begin{tabular}{llll}
\hline & Three attributes & Four attributes & Five attributes \\
\hline Mean-RT (SD) & $1577(512)$ & $1593(501)$ & $1565(519)$ \\
$\begin{array}{l}\text { Number of trials } \\
\text { exceeding deadline }\end{array}$ & 0.85 & 1.27 & 1.50 \\
\hline
\end{tabular}

\section{Reaction times: strategies and correlation with accuracy}

The WADD and the TTB strategies differ in their predictions concerning RT (Glöckner \& Betsch, 2008). While according to TTB the RT should depend on whether there is a tie on the most important dimension, according to $W A D D$ the RT should depend on the absolute difference in the alternatives' weighted averages ( $\triangle \mathrm{WA})$. To test this prediction, we applied to the log-RT-data of each participant (we used log-RT in order to normalize the otherwise skewed values in the RT-distribution that may involve outliers; see also Glöckner \& Betsch, 2008) a multiple linear regression with two factors: (i) $\Delta \mathrm{WA}$, (ii) a binary predictor of a tie on the most important dimension (i.e., the most important attribute; see Table 1 for an example on which the tie variable equals 1 and $\Delta \mathrm{WA}=0.9)$. We compared the standardized regression coefficients for the participants who were classified as $W A D D$ users and those classified as TTB users. As predicted, the difficulty coefficient was stronger for the WADD users $(M=-0.43, S D=$ $0.12)$ compared with the $T T B$ users $(M=-0.32, S D=$ $0.12 ; t(67)=3.56, p<.001$; Fig. 3 , left), while the tie coefficient was higher in magnitude for the TTB users $(M=$ $0.06, S D=0.14)$ compared with the WADD users $(M=$ $0.02, S D=0.09 ; t(31.8)=2.31, p=.027$; Fig. 3, right $)$. Unlike for the TTB users, for the WADD users the tiecoefficient was not significantly different from zero.

\section{Computational-models of strategy choice: beyond the trembling hand}

While the trembling-hand classifications appear to have some validity, as they are supported by differences in subjective weights (Fig. 2) and in RTs (Fig. 3), there are a number of reasons to suspect that these classifications are a simplification and that the participants vary in a more continual, non-dichotomous, manner. First, the weights are subject to individual 
Table 3 Number of participants classified as users of each one of the three strategies (WADD, TTB, EQW-TTB) as a function of the number of attributes

\begin{tabular}{llll}
\hline \multirow{2}{*}{ Strategy } & \multicolumn{2}{l}{ Number of attributes } \\
\cline { 2 - 4 } & Three & Four & Five \\
\hline WADD & 15 & 14 & 17 \\
TTB & 7 & 9 & 7 \\
EQW-TTB & 3 & 2 & 1 \\
WADD/TTB* & 1 & 1 & 1 \\
\hline
\end{tabular}

$W A D D / T T B^{*}$ represents cases in which these two strategies had equal probabilities

variability even within a strategy group, indicating continuity rather than dichotomous strategies. Second, even for participants classified as $T T B$, we obtain a $W A D D$ component in the RT-regression (Fig. 3). Finally, as recently discussed by Hilbig and Moshagen (2014), the trembling-hand type of error is not well matched with the natural assumptions of a WADD model, according to which choice-problems with lower $\triangle W A$ are expected to have more errors. In order to extend the strategy classification to address these issues, we examined a number of computational models and carried out model-comparison using the aggregate Akaike Information Criterion (AIC; Akaike, 1973) and Bayesian Information Criterion (BIC; Schwarz, 1978). As there are only six EQW classifications in our data (out of 78), we discard these and focus on contrasting between $W A D D$ and $T T B$.

Three new models were examined: (i) A probabilistic model, which in each trial deploys $W A D D$ with probability $p$ and TTB with probability $(1-p)$. While this model assumes a trembling-hand error (as before), $\mathrm{p}$ provides a continuous measure of the degree of $W A D D$ use. (ii) $\mathrm{A}$ model that is like (i) with the exception that the WADD errors are not due to a trembling hand assumption, but rather are assumed to reflect Gaussian fluctuations in the $W A D D$ estimation (with zero mean and whose SD is a new model parameter); we still have a trembling hand parameter for errors of the TTB heuristic (see Lee \& Newell, 2011;

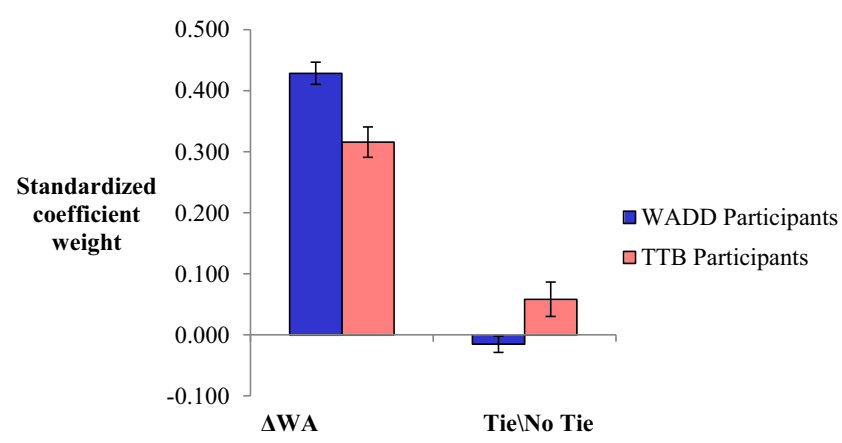

Fig. 3 Averaged standardized regression coefficients of: left ( $\triangle W A-$ difficulty) - for graphic purposes we plotted the negatives of the difficulty-coefficients, and right (TielNo Tie) - whether there was a tie on the most important dimension, separately for participants classified as users of $W A D D$ and $T T B$ strategies. Error bars represent standard errors

Scheibehenne, Rieskamp, \& Wagenmakers, 2013; for similar approaches to mixture models in decision-making). (iii) A fully compensatory model, whose weights are characterized by a single parameter, $\alpha$, based on normalized $\mathrm{W}^{\alpha}{ }_{i}$, (where $\mathrm{W}_{\mathrm{i}}$ are the normative weights (e.g., 4,3,2,1); note that $\alpha>1$ results in an over-weighting of the high weights and under-weighting of the low weights, as in some version of the PCS; Glöckner et al., 2014). For each model, the parameters were fitted based on the probability of the data given the model (see Supplementary Material). The results are summarized at the group level in Table 4 (see Supplementary Material for individual participants' classifications).

We observe a clear picture. The single-strategy tremblinghand models provide the worst fit, followed by the compensatory $\alpha$-weight model, and then by the probabilistic strategy mixture $W A D D / T T B$ model. The best model by far is the mixture model with Gaussian $W A D D$ errors. Importantly, the proportion of WADD use in this model shows high consistency among the participants across the number of attributes (see Suppl.). Furthermore, we also find high correlations between the proportion of $W A D D$ use in the probabilistic model of individual subjects and subjective $\alpha$-weights (all $|\mathrm{r}| \mathrm{s}>.75$; $\mathrm{p}<.001$; see Supplementary Material).
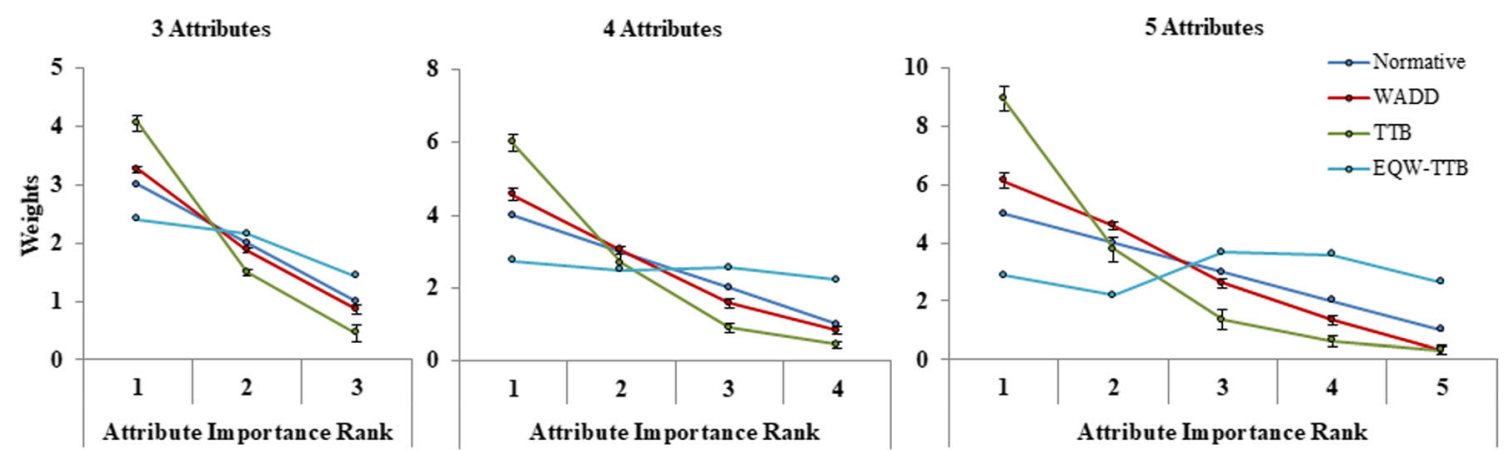

Fig. 2 Subjective weights for jobs with three (left), four (middle), and five (right) attributes, classified by strategy used. Error bars correspond to standard errors 
Table 4 Akaike Information Criterion (AIC) table for models of strategy choice

\begin{tabular}{lllllr}
\hline Model & Pure TTB & Pure WADD & $\begin{array}{l}\text { Binary Mixture Model } \\
\text { (TTB + WADD) }\end{array}$ & $\begin{array}{l}\text { Gaussian mixture model } \\
\text { (TTB + WADD) }\end{array}$ & $\alpha$-weight model \\
\hline Three attributes & 3,941 & 3,135 & 2,566 & $\mathbf{2 , 2 8 3}$ & 2,652 \\
Four attributes & 4,413 & 3,914 & 3,028 & $\mathbf{2 , 6 8 1}$ & 3,241 \\
Five attributes & 4,800 & 4,333 & 3,510 & $\mathbf{3 , 0 9 9}$ & 3,672 \\
\hline
\end{tabular}

Note that AIC differences higher than 10 are considered decisive evidence (bold values indicate the best fits)

a The same conclusions are obtained with BIC (see Tables S8-S10 in Supplementary Material)

\section{Discussion}

In this study, we asked whether participants can rapidly carry out a complex weighted-averaging task. We used a job-interview framing and provided the participants with accuracy feedback. The number of attributes varied from three to five, a range exceeding the capacity of online analytical computations for speedy decisions. The results were surprising. First, the decision times (Mean-RT $\sim 1.5$ $\mathrm{s}$, which includes the visual encoding and the motor response) were much faster than the maximum allotted time, indicating reliance on intuitive gist-perceptions or heuristic rules (Saunders \& Buehner, 2013). Second, despite the short RTs, the accuracy exceeded the bound that could be obtained on the basis of (error-less) non-compensatory strategies, such as TTB. Third, we found a negative correlation between accuracy and decision-time, consistent with evidence integration models, such as those based on the Decision-Field-Theory (Roe et al., 2001), the drift-diffusion model (Krajbich, Armel, \& Rangel, 2010) or PCS (Glöckner et al., 2014). These results are consistent with those obtained by Glöckner and Betsch (2008, 2012) in a multi-cue probabilistic inference task, and with their proposal of an automatic compensatory mechanism.

We examined two simplifying heuristics: The first one (TTB) is non-compensatory, while the second one (EQW-TTB) is compensatory, but neglects the importance of the decision attributes. While the group-level performance exceeded the accuracy bound achievable from an error-free TTB heuristic, at the individual participant level we found a certain amount of variability. The simplified (dichotomous) classification showed that, while most participants relied on compensatory strategies, about $30 \%$ relied on the TTB heuristic. These participants were characterized by a peaked decision-weight pattern that overestimates the most important attribute (Fig. 2), by reduced task accuracy (without a Speed-Accuracy trade-off). TTB-users were also slower in trials with a tie on the most important dimension (Fig. 3, right panel). Unlike TTB users, most participants appeared to deploy a compensatory strategy that is likely to involve a noisy estimation of the weighted average (WADD; see also Glöckner \& Betsch, 2008). The more refined (mixture) classifications indicate a continuum for participants' probability of deploying a compensatory $W A D D$ strategy in each trial, ranging from a minimum of .23 to a maximum of 1 .

We suggest that the presence of variability in decision strategies across the group reflects two potential ways of dealing with time pressure and information overload in decision making. The non-compensatory TTB heuristic is a lexicographic strategy that applies rules sequentially and neglects much of the information. Automatic and compensatory $(W A D D)$ strategies offer an alternative way to deal with information overload. Instead of "calculating" the weighted average, these participants appear to carry out an "approximate" (noisy), but holistic estimation of it, consistent with an affective/intuitive decision mode (Kahneman, 2003). In particular, intuitive/holistic averaging is consistent with Kahneman's suggestion that intuitive processes are holistic in nature (see also Glöckner and Betsch, 2008) and are at the interface of perception and cognition (Kahneman, 2003). This suggestion was also supported by recent empirical results showing dissociations between intuitive and analytical averaging based on load manipulations (Rusou et al., 2017).

A potential mechanism to perform noisy weighted averaging estimations is Glöckner and colleagues' PCS model (Glöckner, Hilbig \& Jekel, 2014). According to this model, the weighted average is computed in a neural network, which multiplies a values-vector with an importance-weights matrix. As our task involves some practice, the assumption that the decision mechanism includes learned weights (reflecting the attributes' importance) is not implausible..$^{2}$ Alternatively, the mechanism of weighted averaging could be mediated by a population code model (Brezis et al., 2016; Brezis et al., 2015; Brezis, Bronfman \& Usher, 2017), which operates using numerosity detectors (Dehaene, Molko, Cohen \& Wilson, 2004; Piazza, Izard, Pinel, Le Bihan, \& Dehaene,

\footnotetext{
2 This does not require to endorse all the assumptions of the $P C S$ model, such as RT being based on convergence to asymptotic activation; an alternative assumption is an integration to boundary. The property of $P C S$ that is important to our results is the parallel integration of values from all attributes. A somewhat similar approach is the accumulator model proposed by Lee and Cummins (2004), according to which the integrated values are subject to a response-boundary. This model, however, assumes that the values are integrated sequentially (in order of importance) and accounts for TTB use for low boundary values. Since in our data TTB users were not faster than WADD users, we support the parallel rather than the sequential integration of values.
} 
2004). Future research is needed to probe the nature of the individual differences underlying the reliance on sequential and holistic processing in multi-attribute decisions.

Acknowledgements We wish to thank Aaron Kravitz for language editing.

Funding Israeli Science Foundation (1413/17) to MU and Binational (Israel-USA) Science Foundation (2014612) to MU.

\section{References}

Akaike, H. (1973). Information theory and an extension of the maximum likelihood principle. In BN, Petrov \& F. Csaki (Eds.), Proceedings of the 2nd International Symposium on Information Theory (pp. 267281). Budapest: Akademiai Kiado.

Brezis, N., Bronfman, Z. Z., Jacoby, N., Lavidor, M. \& Usher, M. (2016). Transcranial Direct Current Stimulation over the parietal cortex improves approximate numerical averaging. Journal of Cognitive Neuroscience, 28(11), 1-14.

Brezis, N., Bronfman, Z. Z. \& Usher, M. (2015). Adaptive spontaneous transitions between two mechanisms of numerical averaging. Scientific Reports, 5, 10415, doi: https://doi.org/10.1038/srep10415

Brezis, N., Bronfman, Z. Z. \& Usher, M. (2017). A perceptual-like population-coding mechanism of approximate numerical averaging. Neural Computation, 30(2), 428-446.

Bröder, A. (2000). Assessing the empirical validity of the "Take-the-best" heuristic as a model of human probabilistic inference. Journal of Experimental Psychology: Learning, Memory, and Cognition, 26(5), 1332-1346.

Bröder, A. (2010). Outcome-based strategy classification. In A. Glöckner \& C. Witteman (Eds.), Foundations for tracing intuition: Challenges and methods (pp. 61-83). New York, NY: Psychology Press.

Cousineau, D. (2005). Confidence intervals in within-subject designs: A simpler solution to Loftus and Masson's method. Tutorials in Quantitative Methods for Psychology, 1(1), 42-45.

Dieckmann, A. \& Rieskamp, J. (2007). The influence of information redundancy on probabilistic inferences. Memory \& Cognition, 35(7), 1801-1813.

Dehaene, S., Molko, N., Cohen, L. \& Wilson, A. J. (2004). Arithmetic and the brain. Current Opinion in Neurobiology, 14(2), 218-224.

Gigerenzer, G. \& Goldstein, D. G. (1996). Reasoning the fast and frugal way: Models of bounded rationality. Psychological Review, 103(4), 650-669.

Gigerenzer, G. \& Goldstein, D. G. (1999). Betting on one good reason: The take the best heuristic. In G. Gigerenzer \& P. M. Todd (Eds.), Simple heuristics that make us smart. Evolution and cognition (pp. 75-95). New York, NY: Oxford University Press.

Glöckner, A. \& Betsch, T. (2008). Multiple-reason decision making based on automatic processing. Journal of Experimental Psychology: Learning, Memory, and Cognition, 34(5), 1055-1075.

Glöckner, A. \& Betsch, T. (2012). Decisions beyond boundaries: When more information is processed faster than less. Acta Psychologica, 139(3), 532-542.

Glöckner, A., Hilbig, B. E. \& Jekel, M. (2014). What is adaptive about adaptive decision making? A parallel constraint satisfaction account. Cognition, 133(3), 641-666.
Hilbig, B. E. \& Moshagen, M. (2014). Generalized outcome-based strategy classification: Comparing deterministic and probabilistic choice models. Psychonomic Bulletin \& Review, 21(6), 1431-1443.

Horstmann, N., Hausmann, D. \& Ryf, S. (2010). Methods for inducing intuitive and deliberate processing modes. In A. Glöckner \& C. Witteman (Eds.), Foundations for tracing intuition: Challenges and methods (pp. 219-237). New York, NY: Psychology Press.

Kahneman, D. (2003). A perspective on judgment and choice: Mapping bounded rationality. American Psychologist, 58(9), 697-720.

Keeney, R. L. \& Raiffa, H. (1976). Decisions with multiple objectives: Preferences and value tradeoffs. New York, NY: Cambridge University Press.

Krajbich, I., Armel, C. \& Rangel, A. (2010). Visual fixations and the computation and comparison of value in simple choice. Nature Neuroscience, 13(10), 1292-1298.

Lee, M. D. \& Cummins, T. D. R. (2004). Evidence accumulation in decision making: Unifying the "take the best" and the "rational" models. Psychonomic Bulletin \& Review, 11(2), 343-352.

Lee, M. D. \& Newell, B. J. (2011). Using hierarchical Bayesian methods to examine the tools of decision-making. Judgment and Decision Making, 6(8), 832-842.

Newell, B. R. (2005). Re-Visions of rationality? Trends in Cognitive Sciences, 9(1), 11-15.

Newell, B. R. \& Shanks, D. R. (2003). Take the best or look at the rest? Factors influencing "one-reason" decision making. Journal of Experimental Psychology: Learning, Memory, and Cognition, 29(1), 53-65.

Oh, H., Beck, J. M., Zhu, P., Sommer, M. A., Ferrari, S. \& Egner, T. (2016). Satisficing in split-second decision making is characterized by strategic cue discounting. Journal of Experimental Psychology: Learning, Memory, and Cognition, 42(12), 1937-1956.

Payne, J. W., Bettman, J. R. \& Johnson, E. J. (1993). The adaptive decision maker. New York, NY: Cambridge University Press.

Piazza, M., Izard, V., Pinel, P., Le Bihan, D. \& Dehaene, S. (2004). Tuning curves for approximate numerosity in the human intraparietal sulcus. Neuron, 44, 547-555.

Roe, R. M., Busemeyer, J. R. \& Townsend, J. T. (2001). Multialternative decision field theory: A dynamic connectionst model of decision making. Psychological Review, 108(2), 370-392.

Rusou Z., Zakay D. \& Usher M. (2017) Intuitive number evaluation is not affected by information processing load. In: J., Kantola, T. Barath, S. Nazir \& T. Andre (Eds.). Advances in human factors, business management, training and education. Advances in intelligent systems and computing 498 (pp. 135-148). Springer International Publishing, Switzerland.

Russo, J. E. \& Dosher, B. A. (1983). Strategies for multiattribute binary choice. Journal of Experimental Psychology: Learning, Memory, and Cognition, 9(4), 676-696.

Saunders, T. S. \& Buehner, M. J. (2013). The gut chooses faster than the mind: A latency advantage of affective over cognitive decisions. The Quarterly Journal of Experimental Psychology, 66(2), 381-388.

Scheibehenne, B., Rieskamp, J. \& Wagenmakers, E. J. (2013). Testing adaptive toolbox models: A Bayesian hierarchical approach. Psychological Review, 120(1), 39-64.

Schwarz, G. (1978). Estimating the dimension of a model. The annals of statistics, 6(2), 461-464.

Tversky, A. (1969). Intransitivity of preferences. Psychological Review, 76(1), 31-48.

Tversky, A. (1972). Elimination by aspects: A theory of choice. Psychological Review, 79(4), 281-299. 